\title{
Fratura traumática da coluna torácica limitada entre T1 e T10
}

\author{
Gibrail Dib Antunes Filho', Jean Roch Alliez¹, Jean Marc Kaya' ${ }^{1}, Y_{v e s ~ R e y n i e r}{ }^{1}, B_{e r n a r d ~ A l l i e z}{ }^{2}$ \\ Serviço de Neurocirurgia - Professor Bernard Alliez. Hospital Nord, AP-HM, Centro Hospitalar Universitário de Marseille, França
}

\section{RESUMO}

Objetivo: Estudo retrospectivo de uma série de 79 pacientes com fratura traumática da coluna torácica, limitada entre T1 e T10, hospitalizados entre 1995 e 2004 no Serviço de Neurocirurgia do Centro Hospitalar Universitário (CHU), Norte de Marseille, França. Métodos: As fraturas foram classificadas de acordo com a classificação da AO (Arbeitsgemeinschaft für Osteosynthesefragen) e o quadro neurológico por meio da classificação de Frankel na hospitalização, aos seis meses e um ano. Resultados: A etiologia mais frequente das fraturas foram os acidentes automobilísticos (68,3\%), e o tipo de fratura, o B (54,4\%); 57 pacientes foram considerados politraumatizados e 82,3\% apresentavam lesão medular. O tratamento cirúrgico foi empregado em $96,2 \%$ dos casos, sendo a via posterior a mais utilizada com objetivo de estabilização, descompressão medular, correção do alinhamento da coluna, diminuição da dor e mobilização precoce. Conclusão: As incidências, as causas, os tipos de fraturas e os manejos destas foram analisados e comparados com a literatura e os resultados confirmaram a gravidade das lesões neste segmento da coluna, o número elevado de lesões associadas, a raridade de recuperação neurológica, assim como o benefício do tratamento cirúrgico por via posterior.

\section{PALAVRAS-CHAVE}

Traumatismos da coluna vertebral entre T1-T10, classificação, tratamento.

\section{ABSTRACT}

Traumatic thoracic spine fractures limited between T1 and T10

Objective: Retrospective study of 79 patients with traumatic thoracic spine fractures limited between T1 and T10, admitted between 1995 and 2004 in the neurosurgical unit of the Marseille University Hospital, France. Methods: Fractures were classified using the AO (Arbeitsgemeinschaft für Osteosynthesefragen) classification and the neurological condition were classified with Frankel's classification at the hospitalization, at six months and one year later. Results: The most frequent etiology of fractures was the motor accidents (68.3\%) and the type B fracture was the most common (54.4\%); 57 patients presented multiple trauma; and $82.3 \%$ presented spinal cord injury. The surgical treatment was employed in $96.2 \%$ of the cases and the posterior approach was the most used. The objectives of the surgical treatment were the spinal stabilization, spinal cord decompression, restoration of a correct spinal alignment, pain reduction and precocious mobilization. Conclusion: The incidences, causes, fractures types and management were analyzed and compared with the literature data. The results confirmed the gravity of the injuries to this segment of the vertebral column, the elevated number of associated injuries, the rarity of neurological recuperation as well as the benefit of the precocious surgical treatment by posterior approach.

\section{KEY WORDS}

Spinal injury at T1-T10 segment, classification, treatment.

\section{Introdução}

As fraturas da coluna vertebral são importante causa de morbidade e de mortalidade na população mundial ${ }^{1}$. As lesões agudas da coluna torácica e da medula espinhal estão entre as causas mais frequentes de incapacidade severa e morte após o trauma ${ }^{3,4}$.
Pouco estudadas na literatura ${ }^{13,28,29}$, as fraturas do segmento superior e médio da coluna torácica (T1 a T10) são, em geral, consideradas como lesões graves geradoras de sequelas neurológicas invalidantes e frequentemente associadas a politraumatismos severos.

Na literatura ortopédica não existe, ainda, um consenso sobre o tratamento (cirúrgico ou ortopédico),

1 Neurocirurgião. Serviço de Neurocirurgia - Professor Bernard Alliez. Hospital Nord, AP-HM, Centro Hospitalar Universitário de Marseille, França. 2 Professor e chefe do Serviço de Neurocirurgia - Professor Bernard Alliez. Hospital Nord, AP-HM, Centro Hospitalar Universitário de Marseille, França. 
sobre o tipo de intervenção (via posterior, anterior ou mista) e também sobre o tempo ideal para a cirurgia nos casos de pacientes politraumatizados.

A fim de simplificar a conduta diante dessa patologia e expor dados clínicos, evolutivos e terapêuticos mais precisos, foi feito um estudo retrospectivo, de uma série de 79 pacientes vítimas de fraturas, exclusivamente traumáticas da coluna torácica, limitadas entre T1 e T10.

\section{Pacientes e métodos}

Foram analisados, retrospectivamente, 79 casos de fraturas da coluna torácica limitada entre T1 e T10, hospitalizados no Serviço de Neurocirurgia do Centro Hospitalar Universitário (CHU), Norte de Marseille, França, entre 1995 e 2004.

Todos os pacientes foram submetidos à tomografia computadorizada (TC) e 31, à ressonância magnética (RM).

Neste estudo, o tipo de fratura foi classificado segundo a classificação da AO (Arbeitsgemeinschaft für Osteosynthesefragen) por ser uma classificação mecanicista das fraturas e basear-se no fato de que a morfopatologia da lesão indica a força ou o momento aplicado sobre o segmento vertebral.

As três forças básicas que produzem as lesões traumáticas do segmento vertebral são:

- Fratura do tipo A: compressão do corpo vertebral.

- Fratura do tipo B: lesão dos elementos anteriores e posteriores por distração.

- Fratura do tipo C: lesão dos elementos anteriores e posteriores com rotação.

O quadro neurológico foi avaliado por meio da classificação de Frankel $^{12}$ no momento da hospitalização do paciente, e a evolução neurológica, comparada com os resultados obtidos em consulta aos 6 meses e 1 ano.

O mecanismo do trauma foi dividido em acidentes automobilísticos (não especificando se passageiro, motorista ou motociclista), acidentes do trabalho e acidentes do esporte ou lazer.

As indicações cirúrgicas e o critério para escolha do método operatório foram baseados no comprometimento do canal medular, instabilidade da coluna, aumento da cifose dorsal e perda da altura do corpo vertebral.

A técnica utilizada, na grande maioria dos pacientes desta casuística, foi a artrodese posterior com parafusos pediculares, com ou sem associação de ganchos pediculares e/ou laminares, sendo a abordagem anterior reservada para os casos de colapso do corpo vertebral e a aborda- gem mista quando a altura do corpo vertebral não tenha sido recuperada com a redução por via posterior.

\section{Resultados}

A média de idade encontrada foi de 38 anos, com extremos de 16 e 81 anos, havendo predomínio do sexo masculino representando $70,9 \%$ (56 casos).

$\mathrm{O}$ espaço de tempo entre o acidente e a hospitalização foi inferior a 6 horas em 40 casos e superior a 6 horas em 39 casos. Os extremos variaram entre 2 horas e 31 dias.

As fraturas foram classificadas de acordo com a sua etiologia: em $54(68,3 \%)$ a causa foi acidente automobilístico; em 23 (29,2\%) foi acidente do trabalho; e em $2(2,5 \%)$, foi acidente esportivo ( 1 acidente de esqui e 1 acidente de escalada).

As fraturas do segmento torácico superior (T1-T5) foram menos frequentes $(42,3 \%)$ quando comparadas às fraturas entre T6 e T10 (57,7\%). No entanto, a porcentagem de pacientes que apresentaram lesões neurológicas foi superior entre T1 e T5 (70\%). A distribuição das fraturas entre $\mathrm{T} 1$ e $\mathrm{T} 10$, resumidas na figura 1 , evidencia uma concentração de fraturas ao nível do pico da cifose dorsal.

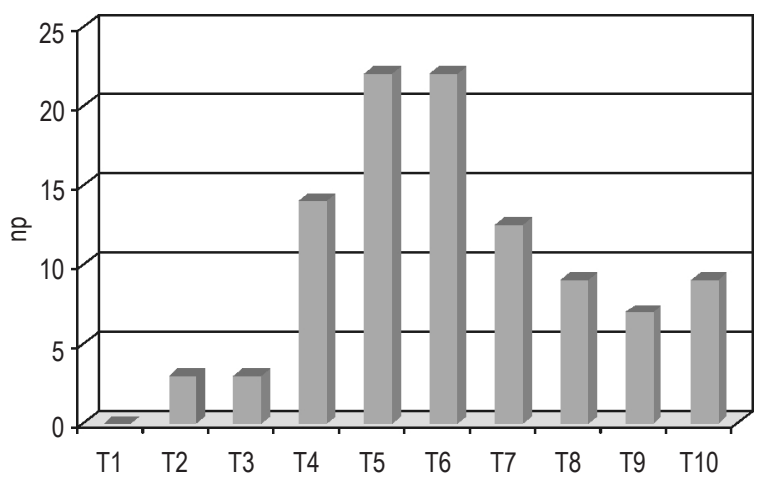

Figura 1-Distribuição das fraturas na coluna torácica.

Entre os 79 pacientes, somente $22(27,9 \%)$ apresentavam fraturas isoladas da coluna torácica. Os 57 pacientes restantes foram considerados politraumatizados por apresentarem lesões em dois sistemas de órgãos, das quais pelo menos uma, ou a combinação das lesões, constituía um risco vital para o doente. Entre as lesões associadas mais frequentes, observamos as torácicas em $53,1 \%$, craniofaciais em $21 \%$, abdominais em $5,7 \%$ e as do esqueleto apendicular em $3,7 \%$ dos pacientes. 
As fraturas por distração, tipo $\mathrm{B}$, foram as mais frequentes $(54,4 \%)$, seguidas pelas fraturas de tipo $\mathrm{A}$ $(32,9 \%)$ e tipo C $(12,7 \%)$.

O quadro neurológico avaliado pela classificação de Frankel na hospitalização dos pacientes mostrou que: $37,9 \%$ apresentavam uma paraplegia sensitivo-motora completa (Frankel A); 44,3\% um déficit neurológico incompleto (14 casos Frankel B, 3 casos Frankel C e 18 casos Frankel D); 17,8\% não apresentam déficits neurológicos (Frankel E).

A análise desses três grupos em relação à classificação da $\mathrm{AO}$ demonstrou que as paraplegias completas são secundárias às fraturas do tipo $\mathrm{B}$ em $23,9 \%$ e ao tipo $\mathrm{C}$ em $11,4 \%$. As paraparesias estavam relacionadas às fraturas tipo $\mathrm{B}$ em $29,1 \%$, tipo $\mathrm{A}$ em $13,9 \%$ e tipo $\mathrm{C}$ em $1,3 \%$.

Observamos também um número significativo de fraturas por flexão-distração posterior com predomínio ligamentar, tipo B1, igual a 31,6\%. Esses valores estão sintetizados na Tabela 1 .

\begin{tabular}{|c|c|c|c|c|c|c|}
\hline Relaçã & itre & oo de & $\begin{array}{l}\text { a } 1 \\
\text { ira }\end{array}$ & défici & rol & \\
\hline Classificação & & gia & & resia & & \\
\hline & & $\mathbf{A}$ & & & & \\
\hline & $\mathrm{N}$ & $\%$ & $\mathrm{~N}$ & $\%$ & $\mathrm{~N}$ & $\%$ \\
\hline A1 & 0 & 0 & 0 & 0 & 3 & 3,8 \\
\hline A2 & 0 & 0 & 0 & 0 & 4 & 5,1 \\
\hline A3 & 2 & 2,5 & 11 & 13,9 & 6 & 7,6 \\
\hline B1 & 10 & 12,6 & 14 & 17,7 & 1 & 1,3 \\
\hline B2 & 7 & 8,8 & 6 & 7,6 & 0 & 0 \\
\hline B3 & 2 & 2,5 & 3 & 3,8 & 0 & 0 \\
\hline $\mathrm{C} 1$ & 3 & 3,8 & 0 & 0 & 0 & 0 \\
\hline $\mathrm{C} 2$ & 5 & 6,3 & 1 & 1,3 & 0 & 0 \\
\hline C3 & 1 & 1,3 & 0 & 0 & 0 & 0 \\
\hline
\end{tabular}

O tratamento empregado foi o cirúrgico na maioria dos casos $(96,2 \%)$. Entre os métodos cirúrgicos utilizou-se a via de acesso posterior com parafusos pediculares e/ou ganchos pediculares e laminares em 72 pacientes; a via de acesso anterior com corporectomia, uso do cage e enxerto ilíaco em 2 pacientes; e o acesso misto, em dois tempos, em 2 pacientes. Nos demais pacientes, com fraturas do tipo A1 e sem déficits neurológicos, foi empregado o tratamento conservador com colete tipo Jewett.

Dos 79 pacientes deste estudo, apenas um apresentou melhora neurológica passando do tipo B para o tipo $\mathrm{C}$ da classificação de Frankel; três pacientes classificados como Frankel A faleceram no decorrer do primeiro ano e os demais permaneceram estáveis (Tabela 2).
Tabela 2

Escore de Frankel na hospitalização, aos seis e doze meses

\begin{tabular}{lccc}
\hline $\begin{array}{l}\text { Escore de } \\
\text { Frankel }\end{array}$ & $\begin{array}{c}\mathbf{N}^{\mathbf{0}} \text { de } \\
\text { pacientes na } \\
\text { hospitalização }\end{array}$ & $\begin{array}{c}\mathbf{N}^{\mathbf{0}} \text { de pacientes } \\
\mathbf{6}^{\mathbf{0}} \text { mês }\end{array}$ & $\begin{array}{c}\mathbf{N}^{\mathbf{0}} \text { de pacientes } \\
\mathbf{1 2}^{\mathbf{0}} \text { mês }\end{array}$ \\
\hline A & 30 & 28 (2 óbitos $)$ & 27 (1 óbito) \\
B & 14 & 13 & 13 \\
C & 3 & 4 & 4 \\
D & 18 & 18 & 18 \\
E & 14 & 14 & 14 \\
Total & $\mathbf{7 9}$ & $\mathbf{7 7}$ & $\mathbf{7 6}$ \\
\hline
\end{tabular}

\section{Discussão}

As fraturas da coluna torácica apresentam aspectos clínicos e evolutivos, em grande parte, condicionados pelas suas características anatômicas. A coluna dorsal integra a caixa torácica que é rígida e restringe em $70 \%$ o movimento da coluna neste segmento, especialmente na extensão do tronco ${ }^{10,30}$. A cifose dessa coluna é limitada por duas lordoses e seus segmentos de transição (cervicodorsal e dorsolombar), que são as zonas de maior fragilidade e mobilidade.

A coluna torácica apresenta um aumento importante da tolerância à compressão pelo fato de as costelas fornecerem força e capacidade de absorção da energia. As fraturas desse segmento da coluna são consequentes a acidentes que apresentam uma potência considerável com impacto e fenômeno de desaceleração importante, o que traduz os tipos das fraturas (54,4\% tipo B), o número e a gravidade das lesões associadas (72,1\% politraumatizados), assim como a incidência de lesão medular (37,9\% de lesão completa e 44,3\% de lesão incompleta) encontrados nesta casuística. Além disso, a medula torácica é mal vascularizada e o canal medular, neste nível, é mais estreito, o que acentua o risco de isquemia medular e uma evolução neurológica desfavorável.

A média etária encontrada (38 anos) nos nossos pacientes é idêntica à da série de Argenson e cols. ${ }^{2}$, mas superior à encontrada nos trabalhos de Krengel e cols. ${ }^{18}$ e Place e cols. ${ }^{24}$, que relataram média de 20 anos. A predominância masculina $(70,9 \%)$ é classicamente citada na literatura, mas inferior aos dados encontrados nestas três publicações (75\% a 78\%).

Assim como ocorre nas outras séries ${ }^{27,28}$, as fraturas observadas em nossos pacientes apresentam uma predominância de lesões associadas $(72,1 \%)$, dominada por traumatismos torácicos $(53,1 \%)$. Os traumatismos cranianos representam $21 \%$, os abdominais, 5,3\% e os do esqueleto apendicular, 3,7\%. Esses resultados diferem dos observados na série de Argenson e cols. ${ }^{2}$, em que os traumatismos cranianos representam $37,1 \%$, 
seguidos pelos traumatismos torácicos $(26,6 \%)$, assim como a série de $\mathrm{Chris}^{6}$, em que a fratura do calcâneo foi a mais comum.

Contrariamente à literatura ${ }^{5,16,26,30}$, na qual os acidentes por queda são mais frequentes, a causa mais comum de fraturas da coluna vertebral nesta série foram os acidentes automobilísticos $(68,3 \%)$, seguidos pelos acidentes do trabalho - caracterizados em sua maioria por quedas de altura $(29,2 \%)$ - e acidentes do esporte $(2,4 \%)$. Esses dados representam, por um lado, a realidade rodoviária da nossa região, assim como o número elevado de hospitalização de vítimas de acidentes automobilísticos, graças ao centro de neurotrauma presente em nosso hospital e a sua localização próxima das principais autoestradas da região. Esses fatos, associados à eficiência do Serviço de Atendimento Móvel de Urgência (SAMU), assim como a utilização frequente do transporte aéreo na remoção dos pacientes, foram responsáveis pela hospitalização de $50,6 \%$ dos pacientes em menos de 6 horas após o acidente.

Entre as várias classificações para as fraturas da coluna torácica ${ }^{7,19}$, utilizamos a da $\mathrm{AO}$, por ser uma classificação mecanicista das fraturas e basear-se no fato de que a morfopatologia da lesão indica a força ou o movimento aplicado sobre o segmento vertebral.

As três forças básicas que produzem as lesões traumáticas do segmento vertebral são: compressão (tipo A), distração (tipo B) e rotação (tipo C). Desse modo, a morfologia da fratura permite a determinação da patogênese da lesão $0^{14}$. Os três tipos básicos de fraturas (A, B e C) são classificados em grupos e subgrupos com base na descrição mais detalhada da morfologia da fratura, permitindo a sua descrição mais precisa. A gravidade da fratura aumenta do tipo A para o tipo C, ocorrendo também esse escalonamento da gravidade da lesão dentro dos grupos e subgrupos. Essa graduação considera também a instabilidade e o prognóstico das lesões e auxilia a escolha do tratamento mais adequado ${ }^{21}$.

Nesta casuística, as fraturas do tipo B corresponderam a $54,4 \%$ e em 25 casos foram causadas por lesão posterior ligamentar (tipo B1), 13 casos por lesão posterior óssea (tipo B2) e 5 casos por lesão anterior em hiperextensão (tipo B3). Essa constatação está de acordo com os mecanismos lesionais de distraçãoflexão, que são os mecanismos de fraturas normalmente causados por acidentes automobilísticos, predominantes nesta série.

Entre as fraturas do tipo A, observamos uma prevalência do tipo A3 (explosão) com 19 casos. Sabemos atualmente que a fratura do tipo compressão é a lesão mais frequente da coluna torácica, sendo identificada em aproximadamente $54 \%$ das fraturas. Ela é provocada, em sua maioria, por quedas e é responsável por um comprometimento nervoso em $47 \%$ dos $\operatorname{casos}^{2,7,22-24}$.
Já as fraturas do tipo $\mathrm{C}$, menos numerosas nesta casuística $(12,7 \%)$ e representadas em 7,6\% dos casos pelo tipo $\mathrm{C} 2$, apresentam uma agressividade importante, sendo responsáveis pela maioria das lesões medulares completas.

A elevada incidência de lesão medular observada neste estudo $(82,2 \%)$ é justificada pela neuroagressividade das fraturas associada às particularidades anatômicas e vasculares desse segmento da coluna e medula.

As fraturas da coluna dorsal não apresentam um consenso terapêutico com relação ao momento ideal para a cirurgia, assim como a técnica utilizada, principalmente se existem lesões associadas. No entanto, a maioria dos autores preconiza a cirurgia de redução e fixação, o mais precoce possível, mesmo sabendo que a descompressão cirúrgica no paciente com lesão medular completa não tem indicação, pois a recuperação neurológica é rara ${ }^{2,13,18,27,33}$.

O objetivo da cirurgia nos pacientes com lesão medular completa foi a estabilização para prevenção de deformidades cifóticas; já nos pacientes sem lesão medular ou com lesão incompleta, o procedimento cirúrgico foi realizado com o intuito de descomprimir o tecido nervoso, corrigir o alinhamento da coluna e estabilizá-la.

A grande maioria dos pacientes desta casuística $(96,8 \%)$ foi operada precocemente (salvo contraindicação em decorrência de lesão associada que colocasse em risco o prognóstico vital). A estabilização precoce da coluna nos pacientes com lesão completa facilitou os cuidados da enfermagem, diminuiu o tempo de hospitalização no setor de reanimação e possibilitou a realização da fisioterapia em melhores condições com diminuição das dores.

$\mathrm{O}$ tipo de montagem que utilizamos neste segmento da coluna, no qual a mobilidade é limitada, foi uma montagem longa (no mínimo dois níveis superiores e inferiores à vértebra fraturada), com repartição harmoniosa das forças em todos os pontos de ancoragem ósseo, que permitiu a restauração do equilíbrio sagital da coluna e evitou um eventual aumento da cifose, assim como a necessidade de reintervenções cirúrgicas. Por outro lado, utilizamos as montagens curtas em zonas de transição, em que a mobilidade deve ser respeitada.

Apesar de ser descrito na literatura ${ }^{6,9,31}$ não haver diferença significativa no resultado entre as fixações por vias anterior e posterior, com relação à cifose, e que existe uma porcentagem elevada $(29,7 \%)$ de complicações cirúrgicas ligadas à via anterior quando comparada à via posterior $(14,1 \%)^{17}$, a abordagem anterior isolada em primeira intenção nos pareceu indicada nos casos em que a deformação cifótica resulte de uma fratura da coluna anterior e média que necessitasse de reconstrução do corpo vertebral ou nos pacientes em que a 
compressão era exclusivamente anterior. No entanto, dois pontos capitais nos parecem merecer uma reflexão: a abordagem da coluna torácica superior é complexa e difícil; a via transtorácica necessita de técnica e material adaptados, justificando, em alguns casos, duas equipes cirúrgicas.

A abordagem mista, em dois tempos cirúrgicos, apresenta $13,7 \%$ de complicações ${ }^{17}$ e foi reservada, em nossa série, aos casos em que existia persistência, após fixação posterior, de uma compressão medular ou uma cifose importante que pudesse comprometer a perenidade da montagem posterior.

\section{Conclusão}

Os resultados observados nesta casuística mostraram uma frequência elevada de lesões medulares e de politraumatizados nos acidentes com fraturas da coluna torácica entre T1 e T10. Confirmaram a raridade de recuperação neurológica nos pacientes que apresentam lesão medular completa e chamaram a atenção ao número elevado de acidentes automobilísticos resultando, principalmente, em fraturas do tipo flexão distração.

Observamos, segundo revisão da literatura, que a via anterior não apresenta vantagens comparadas à via posterior em relação à cifose, além de comportar maior risco cirúrgico.

Demos preferência à cirurgia precoce com o objetivo de dar todas as chances de recuperação aos casos de lesão medular incompleta e possibilitar mobilização rápida, diminuição da dor, fisioterapia precoce e redução do tempo de hospitalização no setor de reanimação nos pacientes com lesão completa. Assim, são importantes o transporte médico rápido e a existência de centros de neurotrauma preparados para receber esses pacientes e possibilitar um tratamento precoce.

\section{Referências}

1. Americam College of Surgeons. ATLS: advanced trauma life support: course for physicians. Chicago;1993.

2. Argenson C, Boileau P, de Peretti F, Lovet J, Dalzotto H. Les fractures du rachis thoracique (T1-T10). A propos de 105 cas. Rev Chir Orthop. 1989;75:370-86.

3. Bohlman $\mathrm{HH}$. Acute fractures and dislocations of the cervical spine: analysis of 300 hospitalized patients and review of the literature. J Bone Joint Surg. 1979;61:1119-42.

4. Bolesta MJ, Bohlman $\mathrm{HH}$. Late complications of cervical fractures and dislocations and their surgical treatment. In: Frymoyer J, editor. The adult spine: principles of practice. New York: Raven Press; 1991. p. 1107-26.
5. Cedeño A, Rangel G, Formigoni M. Fracturas de columna vertebral e inestabilidad post-traumática: experiencia en el Hospital Pérez de Leon: enero 1997 diciembre 1998. Centro Méd. 2000;45:23-31.

6. Chris PC. Acesso anterior versus acesso posterior no tratamento cirúrgico de fraturas torácicas e lombares, Coluna/ Columna. 2006;5:13-18.

7. Denis F. Spine instability as define the three column spine concept in acute spinal trauma. Clin Orthop.1984;189: 65-70.

8. Denis F, Amstrong GWD, Searts K, Matta L. Acute thoracic burst fractures in the absence of neurologic deficit: a comparison between operative and nonoperative treatment. Clin Orthop.1984;189:142-9.

9. Dickman CA, Yahiro MA, Lu HT, Melkerson MN. Surgical treatment alternatives for fixation of unstable fractures of the thoracic and lumbar spine. A meta-analysis. Spine.1994;9(20 Suppl):2266S-73S.

10. El-Khoury GY, Whitten CG. Trauma to the upper thoracic spine: anatomy, biomechanics, and unique imaging features. Am J Roentgenol. 1993;160:95-102.

11. Falavigna A, Righesso Neto O, Ferraz FAP, Boniatti MM. Fratura traumática da coluna torácica T1-T10. Arq Neuropsiquiatr (São Paulo). 2004;62:1095-9.

12. Frankel HL, Hancock DO, Hyslop G, et al. The value of postural reduction in the initial management of closed injuries of the spine with paraplegia and tetraplegia. Paraplegia. 1969;7:179-92.

13. Haid RW, Kopitnik TA. Thoracic fractures: classification and the relevance of instrumentation. Clin Neurosurg. 1992;38:213-33.

14. Helton L, Delfino A. Classificação das fraturas da coluna torácica e lombar. Coluna/Columna. 2002;1:13-22.

15. Howard MP, David H, Courtney WB, Stringer EA. Stabilization of thoracic spine fractures. Resulting in complete paraplegia. Spine. 1994;15:1726-30.

16. Koch A, Graells XSI, Zaninelli EM. Epidemiologia de fraturas da coluna de acordo com o mecanismo de trauma: análise de 502 casos. Coluna/columna. 2006;5:13-8.

17. Knop C, Bastian L, Lange U, Oeser M, Zdichavsky M, Blauth M. Complications in surgical treatment of thoracolumbar injuries. Eur Spine J. 2002;11:214-26.

18. Krengel WF, Anderson P, Henley B. Early stabilization and decompression for incomplete paraplegia due to a thoracic level spinal cord injury, Spine. 1993;18: 2080-7.

19. Louis R. Les theories de l'instabilité. Rev Chir Orthop Reparative Appar Mot. 1977;63:423-5.

20. Madsen PW, Lee TT, Eismont FJ, Green BA. Diagnosis and management of thoracic spine fractures. In: Youmans JR, editor. Neurological surgery. Philadelphia: Saunders; 1996, v. 3, p. 2043-78.

21. Magerl F, Aebi M, Gertzbein SD, Harms J, Nazarian S. A comprehensive classification of thoracic and lumbar injuries. Eur Spine J. 1994;3:184-201.

22. McAfee PC, Yuan HA, Fredrickson BE, Lubicky JP. The value of computed tomography in thoracolumbar fractures: an analysis of one hundred consecutive cases and a new classification. J Bone Joint Surg (Am). 1983;65:461-73.

23. Oliveira PAS, Pires JV, Borges Filho JMM. Traumatismos da coluna torácica e lombar: avaliação epidemiológica. Rev Bras Ortop. 1996;31:771-6.

24. Place HM, Donalson DH, Brown CW, Stringer EA. Stabilization of thoracic spine fractures resulting in complete paraplegia. Spine. 1994;19:1726-30.

25. Regel G, Lobenhoffer P, Grotz M, Pape HC, Lehmann U, Tscherne H. Treatment results of patients with multiple 
trauma: an analysis of 3,406 cases treated between 1972 and 1991 at a German Level I Trauma Center. J Trauma. 1995;38:70-8.

26. Reyes-Sánchez AA. Revisión y analisis de la bibliografía en el tratamiento de las fracturas toracolombares. Coluna. 2003;2:83-92.

27. Schlegel J, Bayley J, Yuan H, Fredricksen B. Timing of surgical decompression and fixation of acute spinal fractures. $J$ Orthop Trauma. 1996;10:323-30.

28. Schweighofer F, Hofer HP, Wildburger R, Stockenhuber N, Bratschitsch G. Unstable fractures of the upper thoracic spine. Langenbecks Arch Chir. 1997;382;25-8.

29. Shapiro S, Abel T, Rodgers RB. Traumatic thoracic spinal fracture dislocation with minimal or no cord injury. Report of four cases and review of the literature. J Neurosurg. 2002;96(3 suppl):333-7.

30. Silberstein B, Rabinovich S. Epidemiology of spinal cord injuries in Novosibirsk, Russia. Paraplegia. 1995;33:322-5.

31. Verlaan JJ, Diekerhof $\mathrm{CH}$, Buskens E, van der Tweel I, Verbout AJ, Dhert WJ, et al. Surgical treatment of traumatic fractures of the thoracic and lumbar spine: a systematic review of the literature on techniques, complications, and outcome. Spine. 2004;29(7):803-14. Review.

32. White AA, Panjabi M. Clinical biomechanics of the spine. Philadelphia: Lippincott; 1990. p.115-86.

33. Yang SC, Yu SW, Chen YJ, Chen WJ. Surgical treatment for thoracic spine fracture-dislocation without neuro deficit. J Formos Med Assoc. 2003;102(8):581-5.

Original recebido em abril de 2008

Aceito para publicação em novembro de 2008

\section{Endereço para correspondência}

Gibrail Dib Antunes Filho

Rua João de Castro, 355/ ap. 32

88501-160 - Lages, SC

E-mails: gibraf@hotmail.com

gibraildib@clinicaneurocor.com.br 